\section{Age-related deficits of manual grasping in a laboratory versus in an everyday-like setting}

\author{
Otmar Bock, Fabian Steinberg \\ Institute of Physiology and Anatomy, \\ German Sport University, Cologne, \\ Germany
}

\begin{abstract}
This study compared the grasping performance of 24 younger (20-30 years of age) and 24 older subjects (60-70 years of age) in a typical laboratory task (L) where movements were repetitive, externally triggered, purposeless and attention-attracting, and in an everydaylike task (E) where movements were part of a rich behavioral repertoire, internally initiated, purposive and little attended. We registered a wide range of kinematic and force parameters, and calculated their within-subject means and variation coefficients.

Multiple differences emerged between the parameter values in $\mathrm{L}$ and $\mathrm{E}$. Factor analysis reduced them to five independent effects. We also found multiple differences between the two age groups, with seniors responding more slowly and in a more stereotyped fashion. Multiple significant task $\mathrm{x}$ age interactions emerged as well, with age differences being more pronounced in $\mathrm{E}$ than in $\mathrm{L}$.

The latter finding is of practical relevance, since it suggests that age-related deficits in some real-life situations may be underestimated in laboratory research. It also is of theoretical relevance: it indicates that brain regions which are particularly vulnerable to aging may contribute to task $\mathrm{E}$ more than to task $\mathrm{L}$.
\end{abstract}

\section{Introduction}

Grasping is a common daily activity: we use our hand to grasp doorknobs, shirt buttons, handrails and a variety of other objects we want to manipulate or hold on to. It is widely accepted that grasping consists of separable components, a transport component that brings the hand near the item of interest, and a grasp component that preshapes the hand in accordance with the object's size, form and orientation.1-3 Although these components have distinct kinematic properties, they are coupled temporally and spatially to achieve a common functional goal.4-6

Once the fingers have established contact with the object, they apply radial (grip) forces to it, large enough to prevent slipping yet small enough to prevent breaking. They also apply tangential (load) forces to it and thus move it along a desired trajectory. Grip and load forces are closely coupled, leaving but a small safety margin of the grip force over the load force to prevent for slipping. 7,8 Force coupling is highly automatized, but it is flexible enough to allow corrections based on sensory feedback ${ }^{7,9,10}$ and to compensate even for dramatic changes of the gravito-inertial environment. ${ }^{11}$

As we grow older, our sensorimotor abilities deteriorate. Elderly subjects move with a longer latency, lower speed and higher trial-totrial variability than young ones, ${ }^{12-14}$ and their deficits worsen for tasks of increasing complexity. 15 Grasping is not spared: the velocity profile of the transport component is conspicuously skewed in old age since the deceleration phase slows down more than the acceleration phase, ${ }^{16}$ and grip forces are generally higher and less responsive to external perturbations. ${ }^{17}$ From findings like these, one could deduce that the manipulation of objects in daily life is more difficult for elderly than for young people; however, the above data came from typical laboratory studies and may not persist unaltered in everyday life.

Indeed, we have documented before that motor performance in typical laboratory settings may differ from that in everyday-like situations. We found that age-related changes of locomotion observed in the laboratory disappeared when subjects walked in a community park, 18 and that the kinematic and kinetic characteristics of grasping in a typical laboratory task differed from those in a more real-life like task even if the physical constraints on grasping were equal. ${ }^{19}$ We attributed this setting-dependence to the fact that our laboratory and everyday-like tasks differed with respect to repetitiveness, intrinsic versus extrinsic triggering, capture of attention, ecological validity and possibly other factors as well; these differences could result in different patterns of brain activity and thus in different movement characteristics. ${ }^{19}$ With the observed settingdependence in mind, we wondered whether the age-related deficits of grasping reported by earlier laboratory studies may differ from those in real life. Since some brain regions shrink more than others in old age, ${ }^{20}$ we reasoned that the pattern of brain activity for laboratory-type movements may be differently affected by aging than that for everyday-like movements, and seniors' deficits on daily tasks may therefore be either more or less dramatic than laboratory work suggests. To find out, we use a similar grasping paradigm as in our preceding study ${ }^{19}$ and compare the performance of young and elderly subjects.
Correspondence: Otmar Bock, Institute of Physiology and Anatomy, German Sport University, Am Sportpark Müngersdorf 6, 50933 Köln, Germany.

Tel. +49.221.4982.7160 - Fax: +49.221.4982.6790. E-mail: bock@dshs-koeln.de

Key words: Context-dependence, aging, prehension, sensorimotor integration, attention.

Acknowledgements: this work was supported by a Grant from the German Ministry for Economy and Technology, administered through the German Space Agency DLR (50WB0825).

Conflict of interests: the authors report no potential conflict of interests.

Received for publication: 26 August 2011. Revision received: 16 March 2012.

Accepted for publication: 26 March 2012.

This work is licensed under a Creative Commons Attribution NonCommercial 3.0 License (CC BYNC 3.0).

(c) Copyright O.Bock and F. Steinberg 2012

Licensee PAGEPress, Italy

Ageing Research 2012; 4:e7

doi:10.4081/ar.2012.e7

\section{Materials and Methods}

\section{Subjects}

Forty-eight subjects participated, half of them were young (12 females, 12 males, age $25.6 \pm 1.5$ years) and the other half was older (12 females, 12 males, age $65.8 \pm 4.46$ years). All reported to be free of musculoskeletal impairments, diseases of the nervous system and visual deficits except for corrected vision. Those who wore eyeglasses continued to use them during the tests. All subjects lived independently in the community, arrived at the agreed-upon testing site in time, and followed our instructions adequately, which we took as evidence for largely intact cognitive functions. All reported not to have participated in research on grasping or cognition within the last 12 months. An ethical approval for this study was given by the institutional review board of the German Sport University Cologne, and all subjects signed an informed consent statement before participating.

\section{Experimental hardware}

Subjects sat at a table in front of a 17" screen. As illustrated in Figure 1, a cylindric lever of $4 \mathrm{~cm}$ length and $1.5 \mathrm{~cm}$ diameter was located $3 \mathrm{~cm}$ to the right of the screen and 20 $\mathrm{cm}$ above the table. The lever could be moved along a rail downward by $3.5 \mathrm{~cm}$, where a 
mechanical stop was encountered. This downward movement stretched a spring which was just strong enough to bring the lever back to the top when it was released. Lever position was registered by a displacement sensor (Burster ${ }^{\circledR} 8740$ ) and the forces applied to the lever by a $6 \mathrm{df}$ force transducer (ATI ${ }^{\circledR}$ Nano 17), both with a sampling rate of $250 \mathrm{~Hz}$. The lever was covered from above, behind and below by a hood, such that it only could be grasped by thumb and index finger; this ensured that subjects used the precision grip even though we had not instructed them to do so (task E, see below).

A joystick was positioned on the table $32 \mathrm{~cm}$ in front of the screen center, its tip being 12 $\mathrm{cm}$ above the table surface. In consequence, the joystick-lever distance was $36 \mathrm{~cm}$ horizontally and $8 \mathrm{~cm}$ vertically. Six reflecting markers of $6 \mathrm{~mm}$ diameter were attached to the subjects' thumb and index finger by double-sided adhesive tape, and their positions were registered by two Vicon ${ }^{\circledR}$ MX-F20 3D high resolutions infrared cameras (sampling rate: $250 \mathrm{~Hz}$, 1680x1280 pixels).

\section{Experimental procedures}

The experimental paradigm consisted of two tasks, as described in full detail elsewhere. ${ }^{19}$ In task L (laboratory), the joystick was mechanically locked in its central position and subjects were asked to move their hand from the joystick to the lever when a visual target was presented. The target was a yellow dot of $2 \mathrm{~cm}$ diameter, and was displayed in the centre of the screen 20 times at randomly varying intervals of 2-6 second, along with an acoustic beep. Subjects had to grasp the lever with their thumb and index finger, move it down and up again, and then return their hand to the joystick. They were asked to respond quickly and as accurately as possible, and were informed that their responses will be registered and analysed.

In task E (everyday-like), the joystick was unlocked and subjects used it to play a computer game of catching virtual spiders with a cursor. Each game level lasted 10 seconds. Subjects then collected a reward (2 cent per spider caught) by moving joystick and cursor to the center, and then sliding the lever down and up. The latter action added the reward earned in the current level to the displayed reward sum. It also started the next game level, up to a total of 20 levels. To keep the game interesting and absorbing, speed and complexity of spider trajectories increased after every $5^{\text {th }}$ level. To account for generalized slowing in old age, the speed was reduced by about $30 \%$ for elderly subjects. We instructed the participants how to play the game, but didn't tell them exactly how to grasp the lever and didn't inform them that their hand movements will be registered.
Summing up, subjects moved their hand from the same joystick position to the same lever position 20 times in $\mathrm{E}$ as well as in $\mathrm{L}$; the two tasks differed only with respect to the behavioural setting in which the movement was embedded: in task $\mathrm{L}$ it was executed repetitively and for its own sake, was externally triggered, and captured the subjects' attention because of the instructions given by the experimenter. In task E, however, it was part of a meaningful action sequence aimed at a desirable ultimate outcome and was triggered by volition. One half of the subjects from each age group participated in task $\mathrm{L}$ and the other half in task E, to exclude carry-over effects from one task to the other.

\section{Data analysis}

Figure 2 shows a schematic presentation of kinematic and force profiles in one grasping

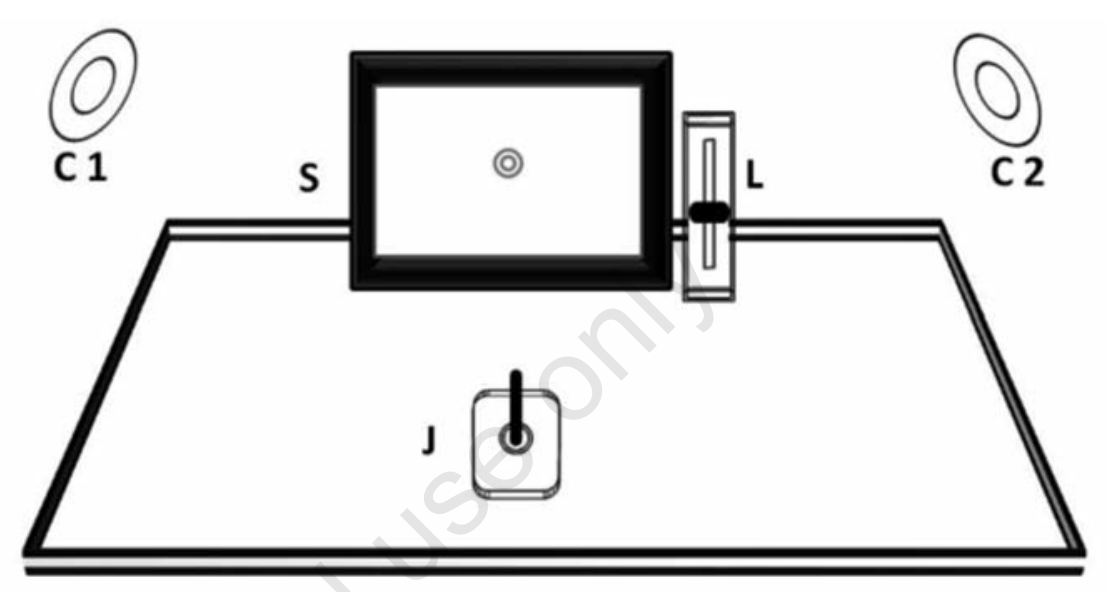

Figure 1. Experimental hardware with table, joystick (J), screen (S), lever (L) and cameras for hand registration $(\mathrm{C} 1 \& \mathrm{C} 2)$. The grey dot on the screen represents the start signal for grasping movements.

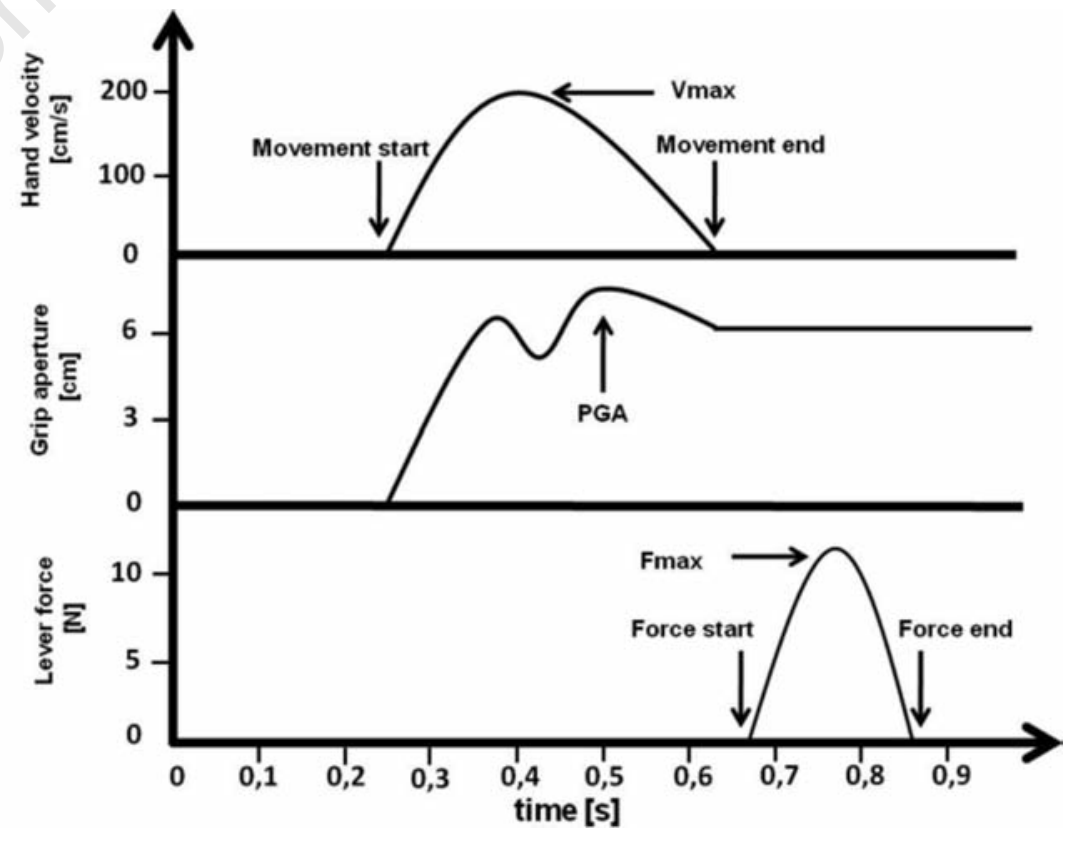

Figure 2. Schematic response profiles in a trial from task $L$. The curves pertain to hand transport (top), grip formation (middle) and lever manipulation (bottom), respectively. The start signal appeared at the time of $0 \mathrm{~s}$. Acronyms refer to parameter names as explained in Supplementary Table 1. 
trial. From such data, an interactive computer routine determined 19 parameters representing the transport component, the grasp component and lever manipulation, as defined in Supplementary Table 1 . We then calculated the mean value of each parameter across the 20 trials per subject, and the corresponding coefficients of variation (CVs). The resultant 19 means and $19 \mathrm{CVs}$ were each submitted to an analysis of variance (ANOVA) with the between-factors Age and Task.

Parameters that yielded significant ANOVA effects for Task and/or Age*Task were submitted to further analyses, to find out whether observed task differences can be reduced to a smaller number of factors. As a first step, we calculated for each parameter the task difference, by subtracting the mean value of that parameter in task L from individual subjects' values in task E and, respectively, by subtracting individual subjects' values in task $\mathrm{L}$ from the mean value in task E. Thus, positive values denote higher scores in task $\mathrm{E}$ than in task $\mathrm{L}$.

Data from young and those from older subjects were normalized separately. The resultant normalized scores differed between age groups, thus reflecting the observed ANOVA effects of Age*Task (see Results). To prevent this difference between group means from confounding the correlations between individual

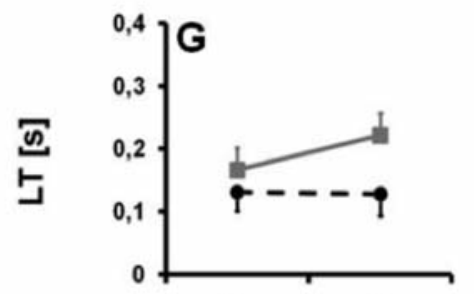

L $\quad$ E
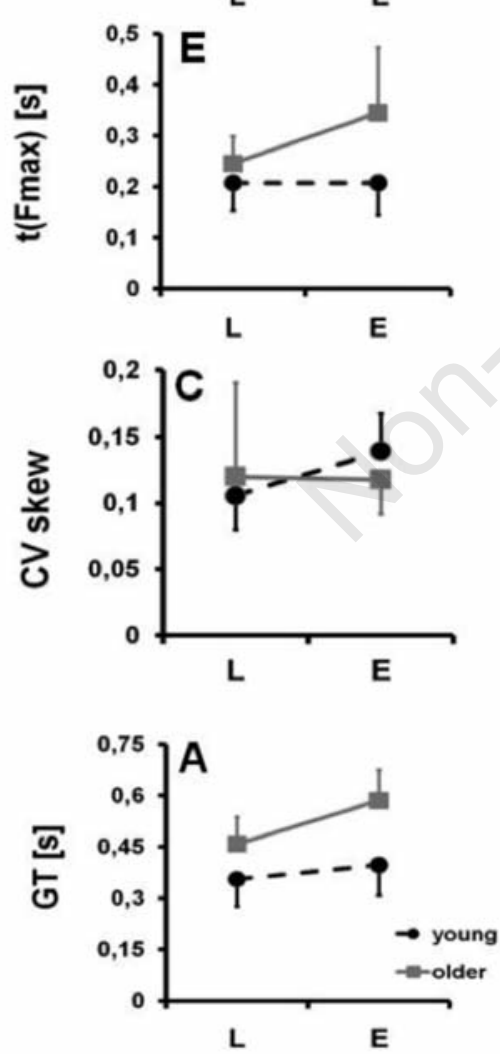
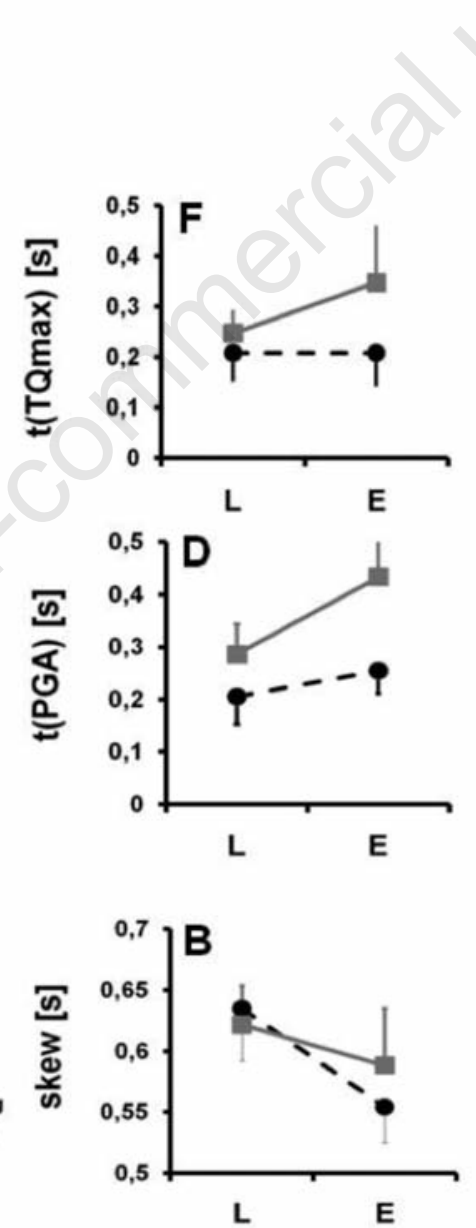

Figure 3. Interaction plots for the seven parameters where interaction was significant. Symbols indicate across-subjects means, and brackets represent the corresponding standard deviation.

scores, we calculated as a second step the differences between each normalized score and the average normalized score for the given task and age group. We then submitted the outcome to a factor analysis with standardized Varimax rotation and a minimum eigenvalue of 1.00 .

\section{Results}

Young subjects earned $5.66 \pm 0.43 €$ and older subjects earned $4.10 \pm 0.60 €$ in the spider-chasing game. Although seniors' earnings were significantly lower $(\mathrm{t}(22)=-7.38$; $\mathrm{P}<0.001$ ) they played surprisingly well, given that spiders speed - and thus the number of spiders they could potentially catch - was $30 \%$ lower than for young subjects.

Supplementary Table 2 summarizes our findings for the 19 parameters representing mean performance, and Supplementary Table 3 for those representing performance variability. The left part of each table shows the acrosssubject averages separately for task L and E, as well as separately for young and elderly subjects, while the right part shows the pertinent ANOVA results (see Supplementary Tables 2 and 3 for $\mathrm{F}$ and P-values). These data reveal several differences between age groups: older subjects' responses were slower than those of young participants in virtually all time-related parameters, their grip aperture was smaller, the hand was more inclined at movement onset, lever forces were higher and response variability was smaller for three but larger for a fourth parameter. Supplementary Tables 2 and 3 also show several differences between tasks: subjects tested with E were slower in virtually all time-related parameters, their grasping movements were more symmetrical (i.e., skew closer to 0.5), the detour and inclination of their hand were larger, lever forces were lower and response variability was larger for four parameters. Finally from Table 2 and 3 , seven parameters yielded significant Task*Age interactions. As Figure 3 illustrates, task-related differences could be less pronounced, more pronounced, or even limited to, the older subject group. It is worth noting in Figure 3 that response slowing in $\mathrm{E}$ was much more pronounced for elderly than for young participants. Eighteen parameters in Supplementary Table 2 and 3 showed significant effects of Task and/or Task*Age, and were therefore selected for factor analysis. Five orthogonal factors emerged, explaining $74.03 \%$ of total variance. The factor loadings are presented in Table 4: factor F1 is related to response speed, F2 to finger-object contact, F3 to the variability of grip force timing, F4 to grip inclination and F5 to hand path curvature. 


\section{Discussion}

Our study compared the grasping movements of young and elderly persons in two settings. Task L was a typical laboratory paradigm: movements were externally triggered, repetitive, attracted subjects' attention (due to the experimenter's instructions) and were executed for their own sake. Task E was designed to approximate typical situations of everyday life as closely as possible: movements were voluntarily initiated, were part of a complex behavioural sequence, were executed to achieve a valid ultimate purpose, and probably attracted little attention as attention was likely to be focussed on the computer game. While the settings were different, the mechanical constraints on grasping were the same for both tasks: movements started from the same joystick, were aimed at the same lever, in the same orientation and location. In consequence, any observed differences between movements in $\mathrm{L}$ and $\mathrm{E}$ should reflect the settings and not physical constraints.

As in our previous study, ${ }^{19}$ we observed multiple differences between $\mathrm{L}$ and $\mathrm{E}$ that affected spatial, temporal and force-related parameters of the transport component, the grasp component and lever manipulation. When age-related differences were averaged out, a factorial structure emerged, with factors reflecting overall movement speed, finger-object contact, transport curvature, variability and hand inclination. The present data therefore indicates that the setting affects at least five independent processes involved in the control of grasping. We also found several differences between age groups. Elderly participants were slower than young ones in all three response components, and their movements were more stereotyped. They tilted the hand more at movement onset, pre-shaped their fingers less for the lever, and established a dramatically higher safety margin early during manipulation. The latter finding is consistent with earlier reports about seniors' higher grip forces, ${ }^{17}$ and could be due to the deficits of force anticipation and proprioceptive feedback in old age.21-24 In accordance with earlier studies on age-related sensorimotor deficits, we interpret our findings as evidence that grasping is impaired in old age, and that part of the deficit is compensated by slowing down. If so, objects designed for daily use by seniors should be particularly resistant to finger impact (collision and compression), and devices that require timed manipulations - such as modern vending machines - should account for seniors' substantially lower response speed.

Earlier studies reported that the deceleration phase of pointing ${ }^{25,26}$ and grasping ${ }^{16}$ slows down more-than-proportionally in old age, such that velocity profiles become asymmetri- cal. This has been attributed to the use of different control strategies and/or to an increased role of feedback-based corrections. ${ }^{16,27}$ However, we found no evidence for a higher asymmetry in the present study, which suggests that the earlier findings are limited to certain movement types. Our subjects grasped a lever that could not be missed or toppled over, which possibly was not much of a challenge even for our elderly participants, and thus didn't require conspicuous adjustments to strategies or feedback use.

Our study also revealed significant Task*Age interactions in seven parameters. The differences between age groups were less pronounced in $L$ than in $E$ for all seven of them, and additionally changed polarity for two parameters. This outcome is both of practical and of theoretical interest. On the practical side, it documents that findings from the laboratory may grossly underestimate the deficits which seniors experience in some situations of everyday life. As an example, grasping time (GT) increased in old age by about $100 \mathrm{~ms}$ in task L, but the increase almost doubled to about $190 \mathrm{~ms}$ in task E. The additional delay of $90 \mathrm{~ms}$ could well be practically relevant. Consider, e.g., a senior who operates an assistive device while walking: if that person stumbles and reaches out to grasp a handrail, $90 \mathrm{~ms}$ could decide whether the reach successfully prevents a fall, or not. As another example, a computerized device such as a mobile phone could allow the user to enter information within a given time window, and that window could be some $90 \mathrm{~ms}$ too short for elderly users. At a theoretical level, the observed Task*Age effects suggest that the control mechanisms for task L and task E are differentially affected by aging. This would fit well with the finding that different brain regions shrink in old age at a different rate, ${ }^{20}$ if we accede that the regions particularly affected by shrinkage make a larger contribution in task $\mathrm{E}$ than in task L. Further research, using electroencephalography, brain imaging or brain-damaged subjects, will be necessary to scrutinize this view.

Finally several qualifying comments should be made regarding task E. First, it should be regarded not as a prototype, but rather as one possible exemplar of an everyday setting: we all execute grasping movements in real life under a wide range of circumstances, some of which may substantially differ from task E, and additional work will be needed to determine exactly which aspect of the task setting determines which characteristic of grasping. Second, the spider-chasing game of task E might be more familiar to young than to elderly subjects, since youngsters are more likely to play joystick-driven computer games; our findings therefore do not necessarily apply to seniors' performance in everyday settings such as household chores and grocery shopping, and rather may be limited to everyday settings such as automated banking, use of assistive devices, and life in intelligent homes

\section{References}

1. Jeannerod M. Intersegmental coordination during reaching at natural visual objects. In: Long J, Baddeley A. Attention and Performance. Hillsdale, NJ: Lawrence Erlbaum; 1981. p. 153-168.

2. Gentilucci M, Chieffi S, Scarpa M, Castiello U. Temporal coupling between transport and grasp components during prehension movements: effects of visual perturbation. Behav Brain Res 1992;47:7182.

3. Castiello U, Bennett K, Chambers H. Reach to grasp: the response to a simultaneous perturbation of object position and size. Exp Brain Res 1998;120:31-40.

4. Haggard P, Wing A. Coordination of hand aperture with the spatial path of hand transport. Exp Brain Res 1998;118:286-92.

5. Rand K, Stelmach, GE. Effect of orienting the finger opposition space in the control of reach-to-grasp movements. J Motor Behav 2005;37:65-78.

6. Marteniuk RG, Leavitt JL, MacKenzie CL, Athenes S. Functional relationships between grasp and transport components in a prehension task. Hum Movement Sci 1990;9:149-76.

7. Johansson RS, Cole KJ. Sensory-motor coordination during grasping and manipulative actions. Curr Opin Neurobiol 1992;2:815-23.

8. Flanagan JR, Tresilian JR. Grip-load force coupling: a general control strategy for transporting objects. J Exp Psychol Hum Percept Perform 1994;20:944-57

9. Haggard P, Wing A. Coordinated responses following mechanical perturbation of the arm during prehension. Exp Brain Res 1995;102:483-94.

10. Rand MK, Shimansky Y, Stelmach GE, Bloedel JR. Adaptation of reach-to-grasp movement in response to force perturbations. Exp Brain Res 2004;154:50-65.

11. Hermsdorfer J, Marquardt C, Philipp J, et al. Moving weightless objects. Grip force control during microgravity. Exp Brain Res 2000;132:52-64.

12. Welford AT. Reaction time, speed of performance, and age. In: Joseph JA. Central Determinants of age-related declines in motor function. New York: Blackwell Publishing Ltd; 1988. p. 1-17.

13. Ketcham CJ, Seidler RD, Van Gemmert AWA, Stelmach GE. Age-related kinematic differences as influenced by task difficulty, target size, and movement amplitude. J 
Gerontol B Psychol Sci Soc Sci 2002;57: P54-P64.

14. Salthouse TA. Speed of behavior and its implications for cognition. In: Birren JE, Schaie KW, editors. Handbook of the psychology of aging (2nd ed.). New York, NY, US: Van Nostrand Reinhold Co; 1985. p. 400-26.

15. Cerella J. Information processing rates in the elderly. Psychol Bull 1985;98:67-83.

16. Weir P, Mallat BJ, Leavitt JL, Roy EA. Macdonald, JR. Age-related differences in prehension: The influence of task goals. J Motor Behav 1998;30:79-89.

17. Cole K, Rotella D. Old age affects fingertip forces when restraining an unpredictably loaded object. Exp Brain Res 2001;136:53542.

18. Bock 0, Hagemann A. An experimental paradigm to compare motor performance under laboratory and under everyday-like conditions. J Neurosci Meth 2010;193:248.

19. Bock 0, Beurskens R. Changes of locomotion in old age depend on task setting. Gait Posture 2010;32:645-9.

20. Raz N. Aging of the brain and its impact on cognitive performance: Integration of structural and functional findings. In: Craik F, Salthouse T, editors. The Handbook of Aging and Cognition. 2 ed. Hillsdale, NJ: Erlbaum; 2000. p. 1-90.

21. Hurley MV, Rees J, Newham DJ. Quadriceps function, proprioceptive acuity and functional performance in healthy young, middle-aged and elderly subjects. Age Ageing 1998;27:55-62.

22. Kaplan FS, Nixon JE, Reitz M, et al. Agerelated changes in proprioception and sensation of joint position. Acta Orthop
1985;56:72-4.

23. Lord SR, Rogers MW, Howland A, Fitzpatrick R. Lateral stability, sensorimotor function and falls in older people. J Am Geriatr Soc 1999;47:1077-81.

24. Flanagan JR, Bowman MC, Johansson RS. Control strategies in object manipulation tasks. Curr Opin Neurobiol 2006;16:650-9.

25. Roy EA, Winchester T, Weir P, Black S. Age differences in the control of visually aimed movements. Journal of Human Movement Studies 1993;24:71-81.

26. Pratt J, Chasteen AL, Abrams RA. Rapid aimed limb movements: age differences and practice effects in component submovements. Psychol Aging 1994;9:325-34.

27. Bennett KMB, Castiello U. Reach to grasp: changes with age. J Gerontol B Psychol Sci Soc Sci 1994;49:P1-P7. 